\title{
Modulation of microwave radiation in the process of resonant interaction with a counter-propagating rectilinear electron beam
}

\author{
I.V. Zotova $^{1}$, N.S. Ginzburg ${ }^{1}$, A.S. Sergeev ${ }^{1}$, A.E. Fedotov ${ }^{1}$, V.Yu. Zaslavsky ${ }^{1,2}$ \\ ${ }^{1}$ Institute of Applied Physics RAS, N.Novgorod, Russia, zotova@appl.sci-nnov.ru \\ ${ }^{2}$ N.Novgorod State University, N.Novgorod, Russia
}

The need for powerful pulsed sources of millimeter and sub-millimeter radiation is motivated by a large number of fundamental problems and practical applications, including diagnostics of plasma, photochemistry, biophysics, new locating systems, and the spectroscopy of various media. Formation of periodic sequences of ultrashort pulses can be achieved based on different methods. For example in [1] the optically controlled switches are realized based on induced photoconductivity effect in semiconductor elements implemented in resonant system. In [2] for the same purpose the method of passive mode locking in electron oscillators with a saturable absorber in the feedback loop is developed.

At the same time, as it was shown in [3], there is an alternative method of periodic ultrashort pulse generation which is based on the cyclotron resonance interaction of power steady-state signal with counter propagating initially rectilinear electron beam. In such system incident stationary signal splits into sequence of short pulses, each of them can be considered as a soliton which propagates in the interaction space without profile change. This paper is devoted to the detail theoretical analysis of described method of short pulses generation with a view to experimental realization of this effect in millimeter waveband.

\section{Basic Equations}

Let us consider the resonance interaction of an initially rectilinear annular electron beam guided by a homogeneous magnetic field $\vec{H}=\vec{z}_{0} H_{0}$ with an electromagnetic wave (Fig. 1) in the cylindrical waveguide with a radius $R$ near the cut-off frequency $\omega_{c}$. Presenting the transverse electric field of the wave in the form $\vec{E}=\operatorname{Re}\left(A(z, t) \vec{E}_{s}(r) e^{i \omega_{c} t-i m \phi}\right)$, where $A(z, t)$ is the complex slowly varied amplitude, $\vec{E}_{S}\left(\vec{r}_{\perp}\right)$ describes the radial structures of the $T E_{m n}$ waveguide mode, we can describe the wave-beam interaction by the set of equation which includes a parabolic equation for the field amplitude combined with averaged electron motion equations:

$$
\begin{aligned}
& \frac{i \sqrt{G}}{4 \beta_{\| 0}^{2}} \frac{\partial^{2} a}{\partial Z^{2}}+\frac{\partial a}{\partial \tau}=p \\
& \frac{\partial p}{\partial Z}+\frac{\partial p}{\partial \tau}+i p\left(\Delta+|p|^{2}\right)=-a .
\end{aligned}
$$

Here the following normalized variables are used:

$$
\begin{gathered}
\tau=\frac{\sqrt{G} \omega_{c} t}{2}, Z=\frac{\sqrt{G} \omega_{c} z}{2 V_{\| 0}}, a=\frac{e A J_{m-1}\left(\kappa R_{0}\right)}{G^{3 / 4} m c \omega_{c}}, \\
\hat{p}=\frac{p_{x}+i p_{y}}{G^{1 / 4} m c} e^{-i \omega_{c} t}, \quad G=\frac{4 e I J_{m-1}^{2}\left(\kappa R_{0}\right)}{m c^{3} \beta_{\| 0}\left(v^{2}-m^{2}\right) J_{m}^{2}(v)}, I \text { is the } \\
\text { electron current, } \kappa=\omega_{c} / c, J_{m}(x) \text { is the Bessel func- }
\end{gathered}
$$

tion, $m$ is the azimuthal index of the operating mode, $R_{0}$ is the injection radius, $v$ is the $n$-th root of $J_{m}^{\prime}(x)=0$, $\Delta=2\left(\omega_{c}-\omega_{H}\right) / \sqrt{G} \omega_{c}$ is the initial cyclotron resonance mismatch, $\omega_{H}=e H_{0} / m c \gamma$ is the electron gyrofrequency. It should be noted that, in contrast with the gyrotron theory, the transverse velocity of electrons at the input of interaction space is zero, that's why there is no the averaging over cyclotron phases in the right part of parabolic equation in (1). At the cross-section $Z=0$ the standard reflectionless boundary condition for the field amplitude [4] is used. At $Z=L$ (where $\left.L=\sqrt{G} \omega_{c} l / 2 V_{\| 0}\right)$ where the stationary signal with amplitude $\quad a_{0}$ and a normalized frequency $\Omega=2\left(\omega_{0}-\omega_{c}\right) / \sqrt{G} \omega_{c}$ enters the system we apply the modified boundary condition [5]:

$$
a(L, \tau)+\frac{G^{1 / 4}}{2 \beta_{\| 0} \sqrt{\pi i}} \int_{0}^{\tau} \frac{1}{\sqrt{\tau-\tau^{\prime}}} \frac{\partial a\left(L, \tau^{\prime}\right)}{\partial Z} d \tau^{\prime}=2 a_{i n} e^{i \Omega \tau}
$$

Simulations show that depending on the parameters several regimes of interaction can be observed in this system including stationary regimes of resonance absorption and non-linear bleaching described in $[3,6]$. At the same time there is an area of parameters where stationary regimes became unstable replaced by a periodic or chaotic self-modulation.

Periodic regime presented in Fig. 2 realizes for following parameters $L=4, a_{0}=6, \Omega_{0}=0.53 \beta_{\| 0}=0.28$ $\Delta=-2$ and $G=10^{-4}$. In this case the input stationary signal transforms to a sequences of ultrashort pulses which propagates through the interaction space without transformation of their form, i.e. exhibit behavior typical for solitons. Soliton nature of formed pulses can be demonstrated for $|\Delta|>>1$, when there is a limiting transition to the equations of gyro-BWO. As it was shown analytically in [3] for an initially rectilinear electron beam such equations possess the soluton-like solution for intensity of electromagnetic field.

\section{Results of 3D PIC Simulations}

The possibility of modulation of stationary signal in the process of resonance cyclotron interaction with the backward wave is confirmed by direct 3D simulations based on the PIC-code CST STUDIO SUITE. Simulations were carried out for operating frequency of $30 \mathrm{GHz}$ and following parameters of an electron beam corresponding to once for planning experiments: electron energy of $15 \mathrm{keV}$, electron current of $0.75 \mathrm{~A}$, resonance magnetic field of $11.5 \mathrm{kOe}$. Interaction takes place in a cylindrical 
waveguide with the radius of $0.3 \mathrm{~cm}$ and a length of $12 \mathrm{~cm}$. The input signal with power $30 \mathrm{~kW}$ has the transverse structure corresponding to the $\mathrm{TE}_{11}$ mode. In Fig.3a the time dependence of an output power is shown. It is seen that after interaction radiation represents the periodical train of short pulses with duration of $3 \mathrm{~ns}$ and peak power of $30 \mathrm{~kW}$. The distance between pulses is of $20 \mathrm{~ns}$.

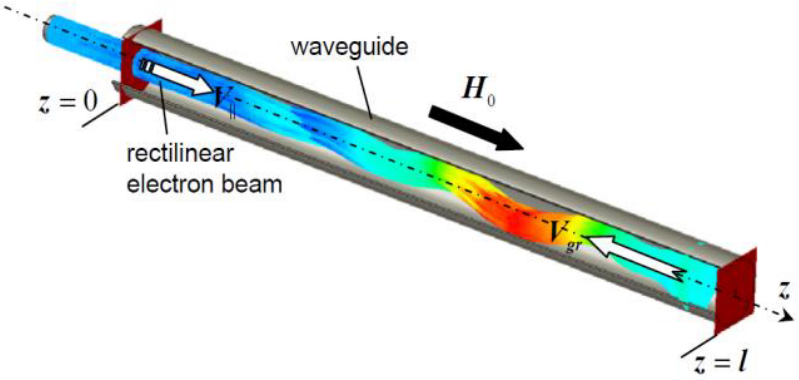

Fig. 1. Scheme of an interaction space with electron trajectories found in PIC simulations

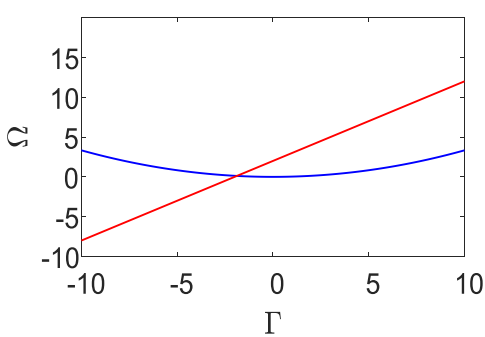

(a)

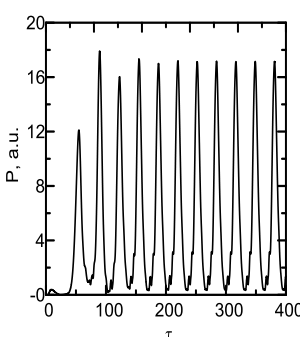

(b)
Fig. 2. Dispersion diagram (a) and formation of periodic sequence of ultrashort pulses (b)

Simulations show that the considered mechanism can be used also for modulation of radiation of short wavelength gyrotrons. In particular, in process of interaction of signal from a $240 \mathrm{GHz} / 350 \mathrm{~W}$ gyrotron [7] with an initially linear annular electron beam having the electron energy $1 \mathrm{keV}$, current $65 \mathrm{~mA}$ and injection radius $0.1 \mathrm{~mm}$ the input stationary signal breaks up into solitons with duration of $0.8 \mathrm{~ns}$ and peak power of $300 \mathrm{~W}$. Due to a significant part of the energy of incident radiation is absorbed by an electron beam the time averaged output power amounts only $30 \%$ from the power of the incident CW signal.

In summary, it was demonstrated that a very simple system consisting of the electromagnetic wave interacting with a counter-propagating beam of initially unexcited oscillators can exhibit a quite complex behavior. The nonlinearity caused by non-isochronous motion of electrons in the magnetic field in combination with time delay effects leads to a nontrivial picture of stationary and nonstationary dynamical regimes. The region of parameters is found in which the incident stationary signal splits into a train of short pulses, and each of them can be interpreted as a soliton.

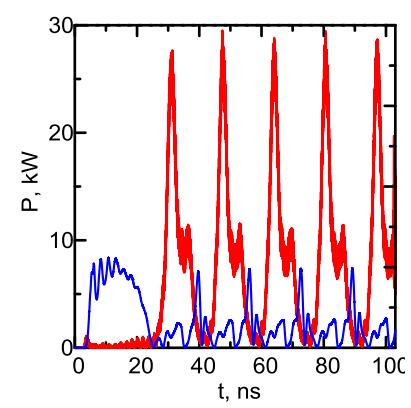

(a)

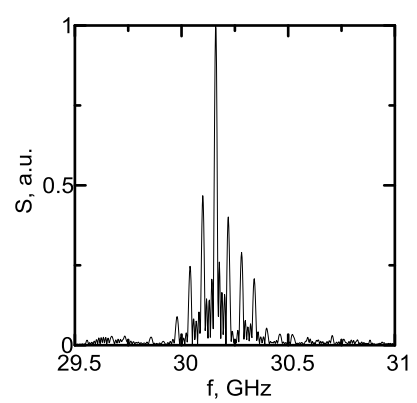

(b)
Fig. 3. PIC simulations of solitons formation for an operation frequency of $36 \mathrm{GHz}$ : time dependence of the output power (a) and radiation spectrum (b). The reflected signal is shown by the blue line

This work was supported by the Russian Foundation for Basic Research (RFBR) (No. 15-08-04506).

\section{References}

1. Vikharev, A.A., Denisov, G. G., Kocharovskiy, V.V., Kuzikov, S.V., Parshin, V.V., Peskov, N.Yu., Stepanov, A.N., Sobolev, D.I., Shmelev, M.Yu. A high-speed quasi-optical wave phase switch based on the induced photoconductivity effect in silicon // Tech.Phys.Lett. 2007. V.33, Iss.9. P.735-739.

2. Ginzburg, N.S., Denisov, G.G., Vilkov, M.N., Sergeev, A.S., Zotova, I.V., Samsonov, S.V., Mishakin, S.V. Generation of trains of ultrashort microwave pulses by two coupled helical gyro-TWTs operating in regimes of amplification and nonlinear absorption // Phys. Plasmas. 2017. V. 24, Iss.2. P. 033101.

3. Zotova, I.V., Ginzburg, N.S., Sergeev, A.S., Kocharovskaya, E.R., Zaslavsky, V.Yu. Conversion of Electromagnetic Wave into a Periodic Train of Solitons under Cyclotron Resonance Interaction with Backward Beam of Unexcited Oscillators // Phys. Rev. Lett. 2014. V.113 P.143901.

4. Ginzburg, N.S. , Nusinovich, G.S., Zavolsky, N.A. Theory of non-stationary processes in gyrotrons with low $\mathrm{Q}$ resonators // Int. J. of Electronics 1986. V. 61, P. 881-894.

5. Ginzburg, N.S., Sergeev, A.S., Zotova, I.V. Time-domain self-consistent theory of frequency-locking regimes in gyrotrons with low-Q resonators // Phys. Plasmas 2015. V. 22, P. 033101.

6. Kovalev, N.F., Kolganov, N.G., Palitsin, A.V., Fuchs, M.I. Proc. of the 4th Int. Workshop "Strong Microwaves: Sources and Applications", N.Novgorod, Russia, 2000, V.2, p. 845.

7. Venediktov, N.P., Dubrov, V.V., Zapevalov, V.E., Kornishin, S.Yu., Kotov, A.V., Kuftin, A.N, Malygin, O.V., Sedov, A.S., Fiks, A.Sh., Tsalolikhin, V.I. Experimental tests of a $263 \mathrm{GHz}$ gyrotron for spectroscopic applications and diagnostics of various media // Radiophys. \& Quant. Electron. 2010. V. 53, Iss.4 P. 237 